Review

\title{
Adopting Intelligent Modelling to Trace Fault in Underground Optical Network: A Comprehensive Survey
}

\author{
Owusu Nyarko-Boateng, Adebayo F. Adekoya and Benjamin A. Weyori \\ Department of Computer Science, The University of Energy and Natural Resources, Sunyani, Ghana
}

\author{
Article history \\ Received: 06-05-2020 \\ Revised: 02-10-2020 \\ Accepted: $13-10-2020$ \\ Corresponding Author: \\ Owusu Nyarko-Boateng \\ Department of Computer \\ Science, the University of \\ Energy and Natural Resources, \\ Sunyani, Ghana \\ Email: owusu.nyarko-boateng@uenr.edu.gh
}

\begin{abstract}
Aiming at building new global optical network infrastructure optimized with fault tracing capabilities, light transmission monitoring, packets re-routing and reconfigurations require an intelligent optical transmission system. An intelligent approach to solve the increasingly complex problems, to enhance fault tracing in the underground optical network infrastructure need to be adopted. For over forty decades now, Optical Time Domain Reflectometry (OTDR) technology has been used to determine faults distance in Fiber Optic Cable (FOC). When it comes to underground optical networking, using OTDR measurements to trace fault on the earth surface takes much longer time since the device only measures the length of underground FOC from the optical transmitter to the point of the fault. Finding the exact spot of fault on earth is a complicated task due to several factors identified in this study. A comprehensive review of previous papers on how OTDR device and other scientific techniques have been used to trace faults in underground FOC were presented in this study. Due to the identified drawbacks in the OTDR to precisely trace fault in underground FOC networks, an intelligent fault tracing technique has been proposed to aid the process. The objective of this paper sought to conduct a comprehensive systematic review of previous studies on fault-finding techniques in underground FOC. To give a clear view of the available technologies used to conduct fault finding activities in underground FOC. The available intelligent systems and the possible future directions of tracing faults in FOC promptly and in a more economical manner.
\end{abstract}

Keywords: Fiber Optics Cable, Artificial Intelligence, Predictive Model, Underground Optical Networks, Machine Learning and OTDR

\section{Introduction}

OTDR has been the widely used device for fault tracing in optical cables for the past four decades (Caballero et al., 2012; Kumar and Rajouria, 2012). When faults occur in underground optical transmission systems, determining the exact spot of the fault on the earth surface is extremely difficult even though the distance of the fault from the optical transmitter is known. In rare cases, optical cables are exposed when faults occur, which may be due to road construction or building projects (Velmuzhov et al., 2018). These cases present an excellent opportunity for the cable maintenance team to identify and resolve the fault (Hayford-Acquah and Asante, 2017) quickly. In a situation where the cables were not exposed but remained underground after a fault occurred, then much effort would be required to trace the fault underground. It takes several hours to dig around and along the optical cable transmission path to identify the spot of the fault. Figure 1 shows the FOC transmission system indicating the distance $\mathrm{X}$, which represents the length of the optical cable from the transmitter to the exact FOC cut or fault point (Kumar and Rajouria, 2012). Y is the distance between the earth surface and the underground FOC. X represents OTDR measurement.

In optical networks and transmission infrastructure, chambers have been mounted at an equal distance along the FOC transmission path. Each chamber houses a certain length of a coiled FOC. The extra coiled cable in the chambers accounts for the difficulty in tracing the exact spot of fault in the underground FOC. The measurement of the OTDR (Kumar and Rajouria, 2012) is insufficient to aid the process of identifying the earth distance. This phenomenon remains one of the major challenges of tracing faults in the underground optical cable network infrastructure. The undue delay in tracing 
faults accounts for long fault repair time and eventually increase revenue loss in the telecommunications industry.

The objectives of this survey sought to conduct a comprehensive review of the previous studies on fault tracing techniques in underground FOC network infrastructure. The survey localized its search on the available intelligent technologies deployed to automate the processes of tracing faults in underground optical cable network infrastructure in a more economical manner. The contribution to the body of knowledge of this paper has been summarized as:

i. A well structured and comprehensive review has been presented on the different techniques and approach used in tracing faults in fiber optics networks

ii. The essential contributions made in the reviewed papers were appropriately critiqued, pointing out the limitations, weaknesses and drawbacks

iii. The identified limitations, weaknesses and drawbacks which characterized the deficiencies in underground FOC fault tracing techniques were duly discussed

iv. A heuristic, intelligent and cognitive optical networks which perceive, act, learn, adapt and optimize the performance OTDR, have been proposed

\section{Structure of the Research Survey}

The survey originally downloaded and reviewed one hundred and five (105) previous works on how OTDR and intelligent modelling have been used to trace fault in optical networks. The papers were selected from high impact factor publications such as Elsevier, Springer, IEEE, OSA and the Wiley. Subsequent to the comprehensive review of the selected papers, we found sixty-four (64) papers most relevant for this work. Fourteen out of the sixty-four papers were directly linked to experimental research using OTDR technology. The remaining papers were also linked to the application of an intelligent system in solving problems associated with the optical network. Figure 2 presents the summary of the artificial intelligence techniques deploying in tracing various faults in optical networks.

Much prominence has not been given to the use of Artificial Intelligence (AI) (Kumar and Rajouria, 2012) to provide solutions to major problems in the underground optical communication network infrastructure. Howevr, huge successes have been achieved in the fault tracing processes in optical communication network infrastructure through the use of AI technology. AI is the modelling of biological and natural intelligence which undergoes through several computations that results in intelligent systems. These intelligent systems include artificial neural networks, evolutionary computation, swarm intelligence, artificial immune systems and fuzzy systems (Seve et al., 2018).

The intelligence system integrates logic, deductive reasoning, expert systems, case-based reasoning and machine learning systems for solving problems through classification, pattern recognition, planning and many others (Eriksson et al., 2017; Potter and Jong, 2000).

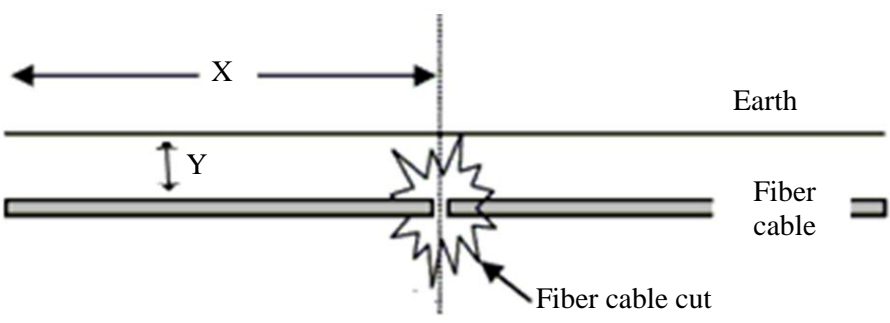

Fig. 1: A cut in fiber optics cable at a distance X, (Kumar and Rajouria, 2012)

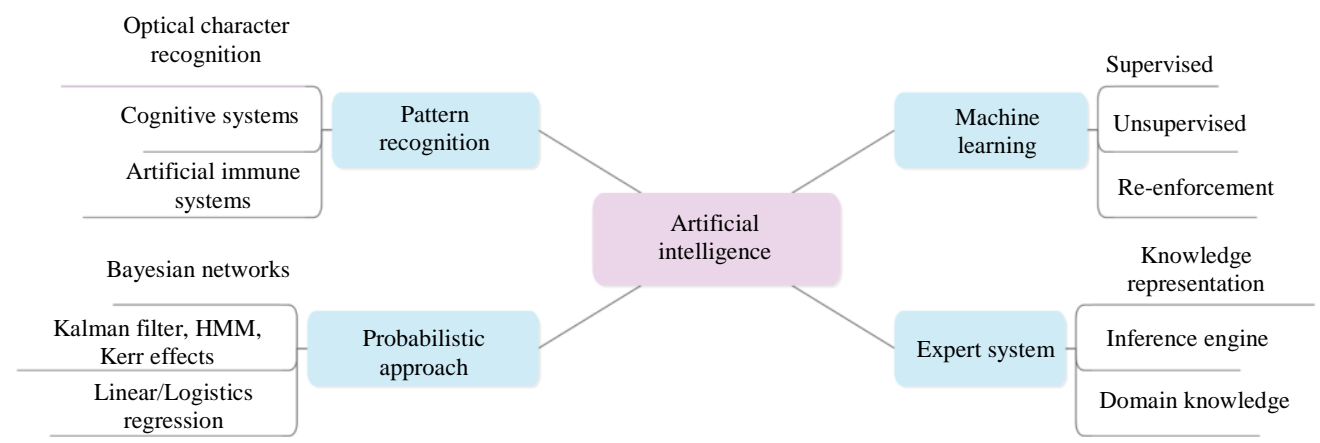

Fig. 2: AI techniques deploying in tracing various faults in optical networks 


\section{Related Works}

The communication system such as voice and data, deployed on fiber optics transmissions infrastructure has revolutionized the telecommunications industry and business in the few decades (Rottondi et al., 2018). Optical communication systems have also been deployed in various private and public organizations to enhance the quality of services they provide (Velmuzhov et al., 2018). Research work on fault tracing in FOC network infrastructure (Urban et al., 2013) has predominately been done by the measurement of the fault distance of the underground FOC using backscattered light principles (Thongdaeng and Worasucheep, 2016). OTDR uses this principle to measure fault distance in fiber optics cables (El-Sayed et al., 2010; Kumar and Rajouria, 2012).

According to research work carried out by (HayfordAcquah and Asante, 2017; Eriksson et al., 2017), through simulations and laboratory works to provide the relevant expected results in fault localization in network systems. Various tools have been used to conduct a series of experiments at laboratories, on the field and in the classroom to achieve exactness of fault tracing (Hayford-Acquah and Asante, 2017). These tools use various scientific principles, instrumentation and measurement techniques to measure distances in fault tracing processes. Previous works on these scientific principles and other techniques include fault tracing using OTDR (Pournoury et al., 2014; Shahkarami et al., 2018), Photon probe fault locator, Raman-based fiber sensors, T-OTDR, Correlation technique utilizing traffic signal, step frequency method (El-Sayed et al., 2010). However, (Kumar and Rajouria, 2012) argued in their paper that fault detection technique which uses OTDR has limitations and drawbacks. The practical approach of determining the fault distance must be focused not only on the measurement of the fault distance of the underground fiber cable but the Euclidean distance on earth surface (Eriksson et al., 2017) from the optical transmitter to the point of the underground cable cut. That notwithstanding, the solution provided by (Kumar and Rajouria, 2012) introduced several cables spliced points along the FOC transmission path, which eventually increased the losses in the underground fiber network (Kyriakopoulos et al., 2016; Tanimura et al., 2016; Zhang et al., 2016).

The OTDR is the main device for measuring fault distance in FOC; it has successfully been used over the years and further research work has been conducted to improve the principle behind its operation such as backscattered light and Fresnel distance, according to (Mas et al., 2005; Urban et al., 2013). The reviewed papers on fault tracing focused mainly on the optical cable, except (Kumar and Rajouria, 2012; Cohen et al.,
2016), who provided the theoretical result on the measurement of the FOC fault distance on the earth's surface (Mo et al., 2018).

The Mean Time To Repair (MTTR) of tracing fault in an underground optical network is high due to the strenuous processes involved. According to (Chen et al., 2006), faults distance in underground fiber optics networks must be identified precisely and accurately on time and spliced correctly to restore interrupted services to maintain high-level customer experience. This can only be achieved if an intelligent system (Mo et al., 2018; Shiryaev et al., 2018; Soujanya et al., 2017; Rubio-Largo et al., 2012) is incorporated in the fault tracing technique to aid in accurate prediction of the fault distance on earth. It is in this regard that this paper has proposed the use of the artificial intelligence model to supplement the functions of the conventional OTDR (Urban et al., 2013). The new model will significantly improve the fault tracing process in the underground optical networks, thereby reducing MTTR and revenue loss of the industry players.

\section{Overview of AI and Related Techniques}

Previous research works have considered five main paradigms of optical network and transmission intelligence systems, namely Artificial Neural Networks (ANN), Evolutionary Computation (EC), Swarm Intelligence (SI), Artificial Immune Systems (AIS) and Fuzzy Systems (FS). These paradigms incorporate intelligence into optical systems and networks on how to handle uncertainty (FS), how to tackle decision-making (EC) and how to learn (ANN), how to optimize a system (SI) and pattern matching ability (AIS) (Eriksson et al., 2017; Shahkarami et al., 2018; Mo et al., 2018).

ANN has been deployed in several optical network systems (Eriksson et al., 2017). ANN is a computational model in ONC which works similar to the functionality of the human nervous system. The intelligent model is implemented based on mathematical operations and a set of parameters required to determine the output of the expected value. Feedforward Neural Network, Radial basis function Neural Network, Kohonen Self Organizing Neural Network, Recurrent Neural Network (RNN), Convolutional Neural Network, Modular Neural Network, time delay NN, Elman and Jordan Simple RNN have been used in various computations to enhance optical network infrastructure (Shiryaev et al., 2018; Mo et al., 2018).

Evolutionary computation is part of AI problemsolving techniques that effectively treat large and complex optimization problems, even in the presence of transmission constraints, Optical Signal to Noise Ratio (OSNR) and dynamic routing environments. EC has 
been deployed in node location, topology design, routing and fault restoration, call admission (Soujanya et al., 2017), wavelength allocation, frequency assignment and dimensioning (capacity assignment). That notwithstanding, some other emerging problems EC could be used to address are ad-hoc networks, node configuration, automated protocol, hardware design, satellite communications, distributed databases and distributed computing (Potter and Jong, 2000).

The use of Swarm Intelligence (Rubio-Largo et al., 2012) in optical network routing, the automation in the optical transmission systems, the growing demand for capacity and the automated network controls have evolved to incorporate complex network optimization operations (Jukan and Chamania, 2017).

Swarm based intelligence systems have also been used to solve network optimization problems, thus, (Kyriakopoulos et al., 2016) published a heuristic method based on ant colony optimization to reduce network energy footprint, whereas (Rubio-Largo et al., 2012) presented a comparative study among three MultiObjective Evolutionary Algorithms (MOEAs) based on swarm intelligence to solve the RWA problem in optical networks (Mata et al., 2018).

Artificial immune network optimization algorithm has been used for the efficient design approach of directional couplers based multiplexers/demultiplexers for optical communication networks (Soujanya et al., 2017; Silva-Santos et al., 2015).

In Fuzzy Systems, complex path evaluation, and dynamic routing using fuzzy logic has provided better results and made decisions faster for data transport in optical networks (Dulik and Cibira, 2015). The fuzzy intelligent system has been used (Seve et al., 2018; Chen et al., 2006; Dulik and Cibira, 2015; Mishra and Bhanja, 2015) in solving transmission optical network impairments over a longer distance. In the course of signal routing, as the transmission distance increases the optical signal undergoes various physical impairments. Physical impairment such as noise generated in optical amplifiers, nonlinear crosstalk appearing in Dense Wavelength Division Multiplexing (WDM/DWDM) systems. This is due to the fiber nonlinearity effects such as Cross-Phase Modulation (CPM) and Four-Wave Mixing (FWM), inter-symbol interference due to optical Chromatic Dispersion (CD) and Polarized Mode Dispersion (PMD), etc. (Mata et al., 2018; Wass et al., 2017).

Machine learning (Buczak and Guven, 2015; Escobar and Morales-Menendez, 2017) is a branch of AI that has also been widely used in optical network infrastructure design, system prediction, descriptive and prescriptive analysis. In machine learning, there are three main functional algorithms, thus, supervised, unsupervised and re-enforcement. Supervised learning deploys techniques such as linear regression, logistic regression, decision trees, artificial neural networks, nearest neighbour models and Support Vector Machines (SVM), etc. This machine learning techniques have been used for optical network performance monitoring, in estimating the Quality of Transmission (QoT) of optical transmission systems (Caballero et al., 2012; Rottondi et al., 2018; Mo et al., 2018) used to allocate resources in data centres (Shiryaev et al., 2018). Unsupervised learning has been applied in clustering and principal component analysis techniques, recognition of modulation layout and transmission impairment mitigation. Reinforcement learning has also been applied learning techniques which include adaptive dynamic programming and TemporalDifference (TD) methods. Markov Decision Process (MDP), light path and wavelength selection are some of the contexts in Optical Burst Switched (OBS) networks (Mata et al., 2018; Wass et al., 2017).

\section{AI Models Applied in Optical Networks Include}

i. Classification - this model aims to predict the class of an input vector (Mishra and Bhanja, 2015; Buczak and Guven, 2015)

ii. Pattern matching - where the aim is to produce a pattern best associated with a given input vector (Kyriakopoulos et al., 2016; Tanimura et al., 2016)

iii. Pattern completion - where the aim is to complete the missing parts of a given input vector (Kyriakopoulos et al., 2016)

iv. Optimization - where the aim is to find the optimal values of parameters in an optimization problem (Jukan and Chamania, 2017; Kyriakopoulos et al., 2016; Tanimura et al., 2016; Rubio-Largo et al., 2012; Dulik and Cibira, 2015)

v. Control - where given an input vector, appropriate action is suggested (Jukan and Chamania, 2017)

vi. Function approximation/time series modelling, index function approximation - where the aim is to learn the functional relationships between input and desired output vectors (Mata et al., 2018)

vii. Data mining/knowledge discovery - to discover hidden patterns from data (Buczak and Guven, 2015; Zhao et al., 2013a)

Much has not been done in the use of AI to localize fault tracing in underground optical network infrastructure (Zhao et al., 2013a). The conventional OTDR technique has been used devoid of an intelligent predictive system. Table 1 summarizes the literature reviewed in the various AI models as applied in optical networks. 
Table 1: AI techniques in the reviewed literature

\begin{tabular}{|c|c|c|c|}
\hline AI Algorithm & AI technique & Application & Literature \\
\hline \multirow[t]{4}{*}{ Neural networks } & Hybrid NN & & $\begin{array}{l}\text { Eriksson et al. (2017; Rottondi et al., 2018; Tanimura et al., } \\
\text { 2016; Mas et al., 2005; Cohen et al., 2016) }\end{array}$ \\
\hline & Recurrent NN & QoT estimation & $\begin{array}{l}\text { Mo et al. (2018; Rubio-Largo et al., 2012; Mata et al., } \\
\text { 2018; Buczak and Guven, 2015) }\end{array}$ \\
\hline & Perceptron & & $\begin{array}{l}\text { Zou et al. (2007; Zhi et al., 2003; Ye et al., 2017; } \\
\text { Thrane } \text { et al., 2016) }\end{array}$ \\
\hline & Support vector machine & & $\begin{array}{l}\text { Wu et al. (2009; García-Pedrajas et al., 2010; Wang et al., } \\
\text { 2016; Musumeci et al., 2018; Engelbrecht, 2007) }\end{array}$ \\
\hline \multirow[t]{2}{*}{ Fuzzy systems } & Simulated annealing & Self-Phase Modulation (SPM) & Dulik and Cibira (2015; Mishra and Bhanja, 2015; \\
\hline & & Cross-Phase Modulation (XPM) & Zhao et al., 2013a; Zhi et al., 2003; Floris et al., 2019) \\
\hline \multirow[t]{5}{*}{$\begin{array}{l}\text { Evolutionary } \\
\text { computation }\end{array}$} & $\begin{array}{l}\text { Evolution algorithm } \\
\text { - Genetic algorithms } \\
\text { - Differential evolution }\end{array}$ & Software-defined network & $\begin{array}{l}\text { Potter and Jong (2000; Shahkarami et al., 2018; } \\
\text { Kyriakopoulos } \text { et al., 2016; Tanimura } \text { et al., 2016; } \\
\text { Rubio-Largo et al., 2012; Mata et al., 2018; } \\
\text { Fernández et al., 2018) }\end{array}$ \\
\hline & $\begin{array}{l}\text { Metaheuristic and SI } \\
\text {-Ant colony optimization } \\
\text {-Particle swarm optimization }\end{array}$ & Dynamic routing & $\begin{array}{l}\text { Mas et al. (2005; Heng et al., 2019; Kovacevic et al., 2018; } \\
\text { Wu et al., 2009; Vela et al., 2017) }\end{array}$ \\
\hline & Brain emotional learning & & Seve et al. (2018; Ezeh and Ibe, 2013; Fernández et al., \\
\hline & Based intelligent controller & & 2018; Wilson, 2012; Temporão et al., 2013; \\
\hline & Darwin machine & & García-Pedrajas et al., 2010; Yuksel et al., 2008) \\
\hline \multirow[t]{5}{*}{$\begin{array}{l}\text { Probabilistic } \\
\text { approach }\end{array}$} & $\begin{array}{l}\text { Bayesian network } \\
\text { Hidden markov model }\end{array}$ & Optical amplification control & $\begin{array}{l}\text { Tanimura et al. (2016; Mas et al., 2005; Wass et al., 2017; } \\
\text { Kovacevic et al., 2018) }\end{array}$ \\
\hline & $\begin{array}{l}\text { Kalman filter } \\
\text { Kerr effect }\end{array}$ & Optical amplification control & Wang et al. (2016; Vela et al., 2017) \\
\hline & Linear/logistics regression & Failure detection and localization & Rottondi et al. (2018; Mata et al., 2018) \\
\hline & Principal component analysis & Modulation format recognition & \\
\hline & Maximum likelihood & Optical amplification control & \\
\hline \multirow[t]{7}{*}{ Pattern recognition } & Optical character recognition & & $\begin{array}{l}\text { Caballero et al. (2012; Jukan and Chamania, 2017; } \\
\text { Zhang et al., 2016; Mo et al., 2018; Soujanya et al., 2017) }\end{array}$ \\
\hline & Cognitive & & $\begin{array}{l}\text { Mata et al. (2018; Silva-Santos et al., 2015; Buczak and } \\
\text { Guven, 2015; Escobar and Morales-Menendez, 2017) }\end{array}$ \\
\hline & Artificial immune system & & $\begin{array}{l}\text { Fernández et al. (2018; Wilson, 2012; Kovacevic et al., } \\
\text { 2018; Vamsi and Rao, 2016) }\end{array}$ \\
\hline & K-means clustering & Flow classification & Floris et al. (2019; Yuksel et al., 2008; Engelbrecht, 2007) \\
\hline & K-nearest neighbor & Automated configurations & \\
\hline & Random forest classifier & & \\
\hline & Decision tree & & Eriksson et al. (2017; Shahkarami et al., 2018) \\
\hline \multirow[t]{5}{*}{ Intelligent agent } & Autonomous agent & Self-Phase Modulation (SPM) & $\begin{array}{l}\text { Caballero et al. (2012; Jukan and Chamania, 2017; } \\
\text { Potter and Jong, 2000) }\end{array}$ \\
\hline & Autonomous planning agent & Automated configurations & \\
\hline & $\begin{array}{l}\text { Control systems } \\
\text {-Hierarchical control system } \\
\text {-Network control system } \\
\text { Distributed AI }\end{array}$ & Cross-Phase Modulation (XPM) & $\begin{array}{l}\text { Rottondi et al. (2018; Mas et al., 2005; Barletta et al., 2017; } \\
\text { Musumeci et al., 2018) }\end{array}$ \\
\hline & Multi-Agent system & Software-defined network & $\begin{array}{l}\text { Caballero et al. (2012; Jukan and Chamania, 2017; } \\
\text { Potter and Jong, 2000) }\end{array}$ \\
\hline & Knowledge-based agent & Software-defined network & Mas et al. (2005; Barletta et al., 2017) \\
\hline
\end{tabular}

\section{Failures in Optical Networks}

Failures in underground fiber optics network infrastructure can be attributed to different causes such as filters shrinking/misalignment (Floris et al., 2019; Zhao et al., 2013b; Yuksel et al., 2008), amplifier malfunctioning and fiber bends. These failures can be categorized into two major types, thus, hard failure and soft failures (Zibar et al., 2015; Yuksel et al., 2008; Barletta et al., 2017):

i. Hard-failures - these are unpredictable failures in the underground FOC. It can be classified as a sudden event. Examples are fiber cuts, power outages, connector failure, etc., (Barletta et al., 2017; Musumeci et al., 2018; Engelbrecht, 2007)

ii. Soft-failures-these failures can be attributed to the gradual transmission degradation due to equipment malfunctioning, filter shrinking or misalignment, etc. These form of failure can be circumvented by performing regular monitoring of Bit Error Rate (BER) test in the optical network until some inconsistencies or anomalies are detected. Early detection of soft-failures helps to prevent service disruption (Rottondi et al., 2018; Vela et al., 2017) 


\section{Fault Detection Mechanism in Fiber Optics Network}

In fiber optics technology, the only known fault which is measurable is the losses in the cable (soft failures), which usually occur due to loss of strength of the light signal in the cable (Rottondi et al., 2018). Another known fault is a cut in the FOC (hard failures), which usually occurs as a result of several activities along the FOC transmission path (Ezeh and Ibe, 2013). Tracing faults in the underground FOC transmission pose severe challenges in the telecommunication industry. OTDR device has mainly been deployed in tracing faults in FOC, but the identified drawback in ODTR is the fact that it measures only the length of the underground FOC without any hint on how to locate the exact spot of fault on the earth's surface. However, the cable repair team encounters enormous difficulty when tracing the exact spot of FOC cut underground; this situation prolongs fault downtime, increase revenue loss and loss of communication services to the users, as asserted by (Hayford-Acquah and Asante, 2017). This paper identified some significant drawbacks in the present approach of tracing faults in FOC.

\section{The Identified Drawbacks in the Existing Fault Tracing Techniques}

The use of OTDR to trace faults in the underground fiber optics network has existed since the inception of the long-distance transmission using the fiber cable. Even though this technique has been used for several decades as a fault tracing mechanism, some significant drawbacks have been identified in its fault tracing processes. These drawbacks have been list as:

i. Excessive delay- there is an extreme delay in pinpointing the exact spot of fault on the earth's surface since the OTDR device measures only the distance of the FOC (Kumar and Rajouria. 2012)

ii. Distance inconsistencies - The length of cable stock in the chambers (coiled FOC) between the optical transmitter and the fault location largely account for the difficulty and distance inconsistencies between the measured value of OTDR and the distance on the earth (Kumar and Rajouria. 2012). Besides, the stock of cable kept in the spliced enclosures prolongs the length of latent length, which OTDR measures but cannot be seen on the earth surface

iii. Waste of resources- the cost of labour for the excessive digging in search of the underground FOC cut point (Hayford-Acquah and Asante, 2017; Nyarko-Boateng et al., 2020a; 2020b)

The use of only OTDR measurement in tracing fault in underground FOC has been a hectic experience over the years (Kumar and Rajouria, 2012). In reality, the distance recorded by the OTDR is always longer than the fault distance on earth (due to the coiled FOC in the chambers and nonlinearity of the underground FOC along the transmission path). As part of the fault tracing processes, the fiber maintenance team searches for the fault location on earth surface along the transmission path. The difficulty in the search arises when the underground fiber cable is not visible. In this case, the repair team practically digs several places along the FOC transmission path in search of the fault spot. This fault tracing approach accounts for delays and waste of resources. After several diggings in an attempt to locate the faulty point, the repair team finds the spot. Historical records on tracing faults in underground FOC transmission has practically been observed that the actual distance of the fault spot on earth has always been less than the OTDR measurement. The factors which account for these irregularities is the extra cable in the chambers.

\section{Tracing Faults in Optical Networks}

The OTDR has been deployed in fault tracing (Mas et al., 2005; Fernández et al., 2018) and assessing splice loss in optical network infrastructure. The device has also been used in most cases to establish the integrity of FOC when it is newly installed (Caballero et al., 2012). The operating principle of the device is based on measurement of Fresnel reflection and the Rayleigh backscattering signal which is generated by sending a high power optical pulse from the OTDR through the optical fiber (Wilson, 2012; Heng et al., 2019). The light reflected back to the OTDR is called backscatter (Pournoury et al., 2014). This backscattered light measured by a sensitive optical receiver in the OTDR is converted to digital waveform and then averaged to improve the Optical Signal to Noise Ratio (OSNR). The resultant signal waveforms presented as a graph called a Trace. The trace is a visual representation of the backscattering coefficient created by the OTDR to determine the activity of the backscattered light. The trace shows the activities on the FOC link such as cuts, splice loss, bends, attenuation and distance of the fault (Zhang et al., 2016; Zou et al., 2007; Addanki et al., 2018).

Fresnel reflection is a discrete reflection which uses the activity of the backscattered light to determine the distance of the light signal, which travels back from the faulty point to the optical transmitter (Wilson, 2012). The fault is caused by a change in reverse coefficient elements such as air gap or severe particles obstructing the free flow of the light signal (Kovacevic et al., 2018). However, by using the information of Fresnel reflection, the OTDR can measure the distance from the optical transmitter to the location of faults (Fernández et al., 2018; Wilson, 2012).

In modelling the backscattered activity in the FOC, this paper discussed Rayleigh and Raman scattering, 
which were the two main scattering approaches adopted in characterizing the faults in FOC. Many researchers, including (Vamsi and Rao, 2016) have used Raman scattering in tracing and measuring the distance of the fault in FOC. When the light of a longer wavelength falls on particles in FOC core, the light scatters at the same wavelength as the incoming light (Rayleigh scattering). However, the wavelength of some of the scattered light changes according to the vibration of the molecules of the particles. This phenomenon is called the Raman Effect. The scattering with a decrease in frequency (an increase in wavelength) of light in the Raman scattering is called the Stokes scattering. On the other hand, the scattering with an increase in frequency (a decrease in wavelength) is called the Anti-Stokes scattering (Vamsi and Rao, 2016; Zhi et al., 2003; Zhou et al., 2014).

Another essential approach that researchers did extensive work on is the use of the Optical Frequency Domain Reflectometry (OFDR) in fault tracing (Ye et al., 2017), the use of Step Frequency Method (SFM) for FOC fault tracing, by separating Rayleigh scattering component from the Fresnel reflection component in an estimation of the fiber impulse response by choosing modulated frequencies. Incoherent-OFDR and CoherentOFDR techniques were used to improve fault tracing in optical networks (Vamsi and Rao, 2016).

A new method for centralized FOC fault tracing (Mas et al., 2005) was presented and experimentally demonstrated by (Temporão et al., 2013). The authors showed in their work by using a tunable OTDR (TOTDR) and by adding wavelength-selective isolators at the output ports of the optical splitter in the remote node, individual OTDR traces (Fernandez et al., 2018; Temporão et al., 2013) for all FOC network branches were obtained by subtraction of traces obtained with two different wavelengths. By using this method, the fault location in FOC could be performed precisely as in pointto-point links (Temporão et al., 2013; Wang et al., 2018).

In an effort to reduce the delays in the underground fault tracing process, (Zibar et al., 2015) experimented an embedded OTDR technique for a cost-efficient FOC monitoring in optical access networks. The authors demonstrated how an embedded OTDR in an Optical Network Unit (ONU) was able to perform better and improve fault tracing in FOC networks. However, (Chen et al., 2006) published a similar work based on (Schmuck et al., 2006) embedded OTDR approach (Zou et al., 2007; Addanki et al., 2018). Tracing fault in underground optical networks is tedious, excessive delays and high economic loss to the industrial players. The adoption of the AI technique to trace faults in the underground optical networks tends to reduce the high impact of the fault significantly.

\section{Application of Intelligent Systems to Trace Faults in Underground Optical Networks}

AI has become the centrepiece of communication (Thrane et al., 2016) and strategic decision making in organizations and it is impacting how swift business processes, demand and supply are reaching the maximum target and profit margins. In the Banking and finance business, AI has been deployed for fraud detection projects, credit risk calculations, automation of frequent customer queries. AI has been used in the Retail Sectors for customer service, expert advice on purchases and operations related to multiple channels. Logistics and transport industry is using AI for self-driving cars, radars to detect obstacles and pedestrians, intelligent search for free parking spaces and route optimization. The insurance sector has deployed machine learning to identify new product trends through customer data, automating tasks and detecting fraud (Mata et al., 2018; Wilson, 2012; Wu et al., 2009).

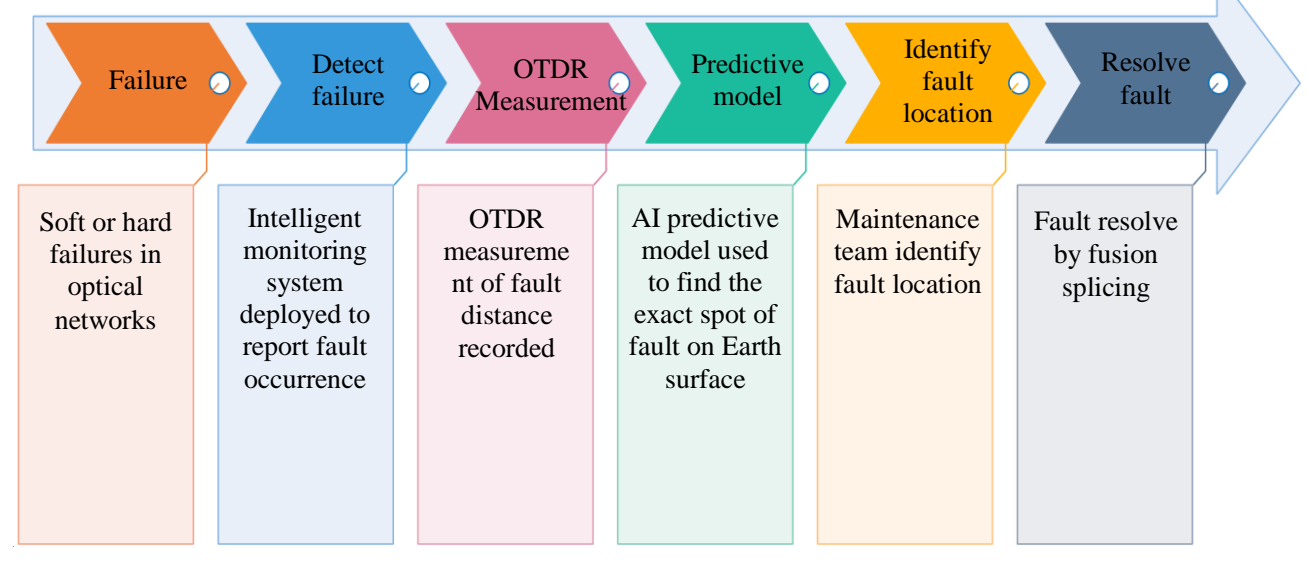

Fig. 3: Application of AI in underground optical network fault tracing 
In telecommunications, AI has been used in signal optimization (Cohen et al., 2016), improved QoT, monitoring (Chen et al., 2006) and Fault detection (Mas et al., 2005; Mata et al., 2018). This paper has localized the application of AI in tracing faults in underground optical network infrastructure (Thrane et al., 2016). Figure 3 shows the processes involved in a model of AI technique to trace fault in underground optical networks. As indicated earlier, since AI has successfully been implemented to solve problems in other areas, tracing fault in underground optical networks cannot exceptional.

\section{Pattern Extraction}

In machine learning, pattern extraction is a technique used to classify the semblance of trained data. The extraction is executed prior to pattern recognition. Pattern recognition is the process of recognizing patterns by using a Machine Learning algorithm. Pattern recognition is the classification of data based on knowledge already gained or on statistical information extracted from patterns representation. Pattern recognition is the ability to detect arrangements of characteristics or data that yield information about a given system or data set (Soujanya et al., 2017; Escobar and Morales-Menendez, 2017).

The data required to produce an excellent result for the proposed AI deployment in tracing fault in the underground optical network may be the OTDR measurement, the previous spliced data and other relevant information. This information represents the input data to the various layers in the predictive model. The input data undergoes through data pre-processing, transformation and pattern extraction process which involves the partitioning of the data into training data and testing data by applying the appropriate machine learning algorithm such as K-nearest neighbour, Decision tree, Random forest, etc. The extracted pattern has to be evaluated to ascertain the performance of the predictive model (Mata et al., 2018; García-Pedrajas et al., 2010; Zeng and Huang, 2004).

\section{Performance Evaluation}

Performance evaluation plays a leading role in the process of predictive modelling. The performance of a predictive model is computed and matched by selecting the appropriate metrics. Choosing the right metrics is essential for a particular predictive model in order to get an accurate outcome (Tamura et al., 2018; Wang et al., 2016; Zibar et al., 2015). It is imperative to evaluate predictive models because various kinds of datasets may be used for the same predictive model. Some of the standard metrics which have been used to evaluate predictive models are Confusion Matrix, ConcordantDiscordant Ratio, Cross-Validation, Gain and Lift Chart,
Kolmogorov Smirnov chart, mean square error, median absolute error, percent correction classification and root mean squared error (Mata et al., 2018). Some performance evaluation matrix has been presented in Equations 1, 2, 3, 4 and 5.

Similarly, performance is the most important aspect that has to be considered when a predictive model is being designed (Mo et al., 2018; Tamura et al., 2018; Wang et al., 2016; Zibar et al., 2015). The performance of the model is not just measured as the accuracy achieved by prediction, but aspects such as computational complexity of the FOC fault splicing history. The design of the model for optimal performance requires careful consideration of several factors that influence the optical network performance (Floris et al., 2019; Zhao et al., 2013b; Yuksel et al., 2008; Barletta et al., 2017).

Most predictive models (Rottondi et al., 2018; Vela et al., 2017; Musumeci et al., 2018; Engelbrecht, 2007) adopted Mean Squared Error (MSE) as a performance evaluation, in which case the training error, $\varepsilon_{T}$, is expressed as:

$$
\varepsilon_{T}=\sum_{p=1}^{P_{T}} \sum_{k=1}^{k}\left(t_{k, p}-o_{k, p}\right)^{2}
$$

where, $P_{T}$ is the total number of training patterns in the training dataset and $k$ is the number of output units. Instead of the MSE, the Sum Squared Error (SSE), can be used, where $P$ is the total number of patterns in the dataset considered. Nevertheless, the SSE is not a good measure when the performance on different dataset sizes are matched:

$$
\text { sse }=\sum_{p=1}^{p} \sum_{k=1}^{k}\left(t_{k, p}-o_{k, p}\right)^{2}
$$

When the ramp or step function is used as the activation function in the output layer, the output of the model is the same as the target. In the case of continuous activation functions, a pattern recognition is considered as been correctly classified for each output unit $O_{k},\left(\left(O_{k, p}\right.\right.$ $\geq 0.5+\theta$ and $\left.t_{k, p}=1\right)$ or $\left(O_{k, p} \leq 0.5-\theta\right.$ and $\left.\left.t_{k, p}=0\right)\right)$, where threshold, $\theta \in[0,0.5]$. An additional measure of accuracy is to compute the correlation between the output and target values for all patterns. The correlation coefficient (Vela et al., 2017) is computed as:

$$
\begin{aligned}
& r=\frac{\sum_{i=1}^{n}\left(x_{i}-\bar{x}\right) \sum_{i=1}^{n}\left(y_{i}-\bar{y}\right)}{a_{x} a_{y}} \\
& =\frac{\sum_{i=1}^{n} x_{i} y_{i}-\frac{1}{n} \sum_{i=1}^{n} x_{i} \sum_{i=1}^{n} y_{i}}{\sqrt{\sum_{i=1}^{n} x_{i}^{2}-\frac{1}{n}\left(\sum_{i=1}^{n} x_{i}\right)^{2}} \sqrt{\sum_{i=1}^{n} y_{i}^{2}-\frac{1}{n}\left(\sum_{i=1}^{n} y_{i}\right)^{2}}}
\end{aligned}
$$




$$
=\frac{\sum_{p=1}^{p} O_{k, p} t_{k, p}-\frac{1}{p} \sum_{p=1}^{p} O_{k, p} \sum_{p=1}^{p} t_{k, p}}{\sqrt{\sum_{p=1}^{p} O_{k, p}^{2}-\frac{1}{p}\left(\sum_{p=1}^{p} O_{k, p}\right)^{2}} \sqrt{\sum_{p=1}^{p} t_{k, p}^{2}-\frac{1}{p}\left(\sum_{p=1}^{p} t_{k, p}\right)^{2}}}
$$

Another important aspect of predictive modelling accuracy is overfitting. Overfitting of a training dataset means that the model memorizes the training patterns and consequently loses the ability to generalize. Thus, the model that overfit cannot predict the correct output for data patterns not seen during training (Yuksel et al., 2008; Barletta et al., 2017; Tanimura et al., 2018). Overfitting occurs when the architecture of the model is too large. The larger the architecture, the more computations are required to predict outputs after training and the more learning computations are needed per pattern presentation (Escobar and Morales-Menendez, 2017). The larger the size of the training dataset, the more patterns are presented for training. Hence, the total number of learning calculations per epoch is increased. However, estimations of generalization error during training can be used to detect the point of overfitting (Eriksson et al., 2017; Mata et al., 2018).

The accuracy of the predictive model has to be measured by the main evaluation metrics indicated. The Mean Absolute Error (MAE) and Root Mean Squared Error (RMSE) are two of the most common metrics used to measure the accuracy of the predicted variable. MAE measures the average magnitude of the errors in a set of predictions whiles RMSE is a quadratic scoring rule which has to be deployed to measure the average size of the error. Both MAE and RMSE expressed average model prediction error which means metrics ranged from 0 to $\infty$ and are indifferent to the direction of errors. The matrics are negatively-oriented scores, which means lower values are better. The Correlation Coefficient (CC) indicates the strength of the relationship between the measured values of the OTDR and the predicted actual value of the underground FOC fault distance (Engelbrecht, 2007).

\section{Conclusion}

The reviewed papers indicate that OTDR measurement for the underground fault-finding presents an extremely cumbersome task. The functionality of the conventional OTDR can be improved by enhancing its operations with the appropriate AI technology. In this comprehensive survey, the authors have proposed an AI model to be incorporated in the OTDR, which will automate the output with the measurement of the exact earth distance. The improved FOC fault tracing with OTDR or the predictive model is an efficient and precise fault location system which tends to reduced fault restoration time significantly, reduce the cost of maintenance and even predict the next possible fault location in the optical network infrastructure.

\section{Acknowledgement}

The publication of this paper was sponsored by the University of Energy and Natural Resources, Sunyani. We are must grateful to the management of the University.

\section{Author's Contributions}

The authors contributed equally to research, reviews and the drafting of this paper.

\section{Ethics}

All the sections, the research organization and all other aspects of this paper were originally written by the authors unless otherwise referenced.

\section{Reference}

Addanki, S., Amiri, I. S., \& Yupapin, P. (2018). Review of optical fibers-introduction and applications in fiber lasers. Results in Physics, 10, 743-750.

Barletta, L., Giusti, A., Rottondi, C., \& Tornatore, M. (2017, March). QoT estimation for unestablished lighpaths using machine learning. In Optical Fiber Communication Conference (pp. Th1J-1). Optical Society of America.

Buczak, A. L., \& Guven, E. (2015). A survey of data mining and machine learning methods for cyber security intrusion detection. IEEE Communications surveys \& tutorials, 18(2), 1153-1176.

Caballero, A., Aguado, J. C., Borkowski, R., Saldaña, S., Jiménez, T., de Miguel, I., ... \& Lorenzo, R. M. (2012). Experimental demonstration of a cognitive quality of transmission estimator for optical communication systems. Optics express, 20(26), B64-B70.

Chen, W., De Mulder, B., Vandewege, J., \& Qiu, X. Z. (2006). Embedded OTDR monitoring of the fiber plant behind the PON power splitter. In IEEE/LEOS Benelux Chapter 2006 (pp. 13-16).

Cohen, E., Malka, D., Shemer, A., Shahmoon, A., Zalevsky, Z., \& London, M. (2016). Neural networks within multi-core optic fibers. Scientific Reports, 6, 29080.

Dulik, M., \& Cibira, G. (2015, November). Fuzzy Logic for Optimized Path Establishment in Optical Networks. In Federated Conference on Software Development and Object Technologies (pp. 54-64). Springer, Cham. 
El-Sayed, M., Ibrahim, P. J., \& Gunzer, F. (2010, December). Investigation of the precision regarding fiber fault location with a commercial Optical Time Domain Reflectometer. In 7th International Symposium on High-capacity Optical Networks and Enabling Technologies (pp. 237-241). IEEE.

Engelbrecht, A. P. (2007). Computational intelligence: an introduction. John Wiley \& Sons.

Eriksson, T. A., Bülow, H., \& Leven, A. (2017). Applying Neural Networks in Optical Communication Systems: Possible Pitfalls. IEEE Photonics Technology Letters, 29(23), 2091-2094.

Escobar, C. A., \& Morales-Menendez, R. (2017, July). Machine learning and pattern recognition techniques for information extraction to improve production control and design decisions. In Industrial Conference on Data Mining (pp. 286-300). Springer, Cham.

Ezeh, G., \& Ibe, O. (2013). Efficiency of optical fiber communication for dissemination of information within the power system network. IOSR Journal of Computer Engineering (IOSR-JCE), 12(3), 68-75.

Fernandez, M. P., Rossini, L. A. B., Pascual, J. P., \& Caso, P. A. C. (2018). DSP-Enhanced OTDR for Detection and Estimation of Events in PONs. arXiv preprint arXiv:1801.06485.

Fernández, M. P., Rossini, L. A. B., Pascual, J. P., \& Caso, P. A. C. (2018). Enhanced fault characterization by using a conventional OTDR and DSP techniques. Optics express, 26(21), 27127-27140.

Floris, I., Sales, S., Calderón, P. A., \& Adam, J. M. (2019). Measurement uncertainty of multicore optical fiber sensors used to sense curvature and bending direction. Measurement, 132, 35-46.

García-Pedrajas, N., Del Castillo, J. A. R., \& OrtizBoyer, D. (2010). A cooperative co-evolutionary algorithm for instance selection for instance-based learning. Machine Learning, 78(3), 381-420.

Hayford-Acquah, T., \& Asante, B. (2017). Causes of fiber cut and the recommendation to solve the problem. IOSR J. Electron. Commun. Eng, 12, 46-64.

Heng, X., Gan, J., Zhang, Z., Qian, Q., \& Yang, Z. (2019). Amplification of orbital angular momentum modes in an erbium-doped solid-core photonic bandgap fiber. Optics Communications, 433, 132-136.

Jukan, A., \& Chamania, M. (2017, July). Evolution towards smart optical networking: Where artificial intelligence (AI) meets the world of photonics. In Photonic Networks and Devices (pp. NeM2B-4). Optical Society of America.

Kovacevic, M. S., Kuzmanovic, L., \& Djordjevich, A. (2018). Estimation of Rayleigh scattering loss in a double-clad photonic crystal fiber. Optical and Quantum Electronics, 50(5), 217.
Kumar, V., \& Rajouria, D. (2012). Fault Detection Technique by using OTDR: Limitations and drawbacks on practical approach of measurement. (IJETAE), 2, 283-287.

Kyriakopoulos, C. A., Papadimitriou, G. I., Nicopolitidis, P., \& Varvarigos, E. (2016). Energyefficient lightpath establishment in backbone optical networks based on ant colony optimization. Journal of Lightwave Technology, 34(23), 5534-5541.

Mas, C., Tomkos, I., \& Tonguz, O. K. (2005). Failure location algorithm for transparent optical networks. IEEE Journal on Selected Areas in Communications, 23(8), 1508-1519.

Mata, J., de Miguel, I., Duran, R. J., Merayo, N., Singh, S. K., Jukan, A., \& Chamania, M. (2018). Artificial intelligence (AI) methods in optical networks: A comprehensive survey. Optical switching and networking, 28, 43-57.

Mishra, D., \& Bhanja, U. (2015). FWM Aware Fuzzy Dynamic Routing and Wavelength Assignment in Transparent Optical Networks. International Journal of Electronics and Communication Engineering, 9(8), 985-994.

Mo, W., Huang, Y. K., Zhang, S., Ip, E., Kilper, D. C., Aono, Y., \& Tajima, T. (2018, March). ANN-based transfer learning for QoT prediction in real-time mixed line-rate systems. In 2018 Optical Fiber Communications Conference and Exposition (OFC) (pp. 1-3). IEEE.

Musumeci, F., Rottondi, C., Nag, A., Macaluso, I., Zibar, D., Ruffini, M., \& Tornatore, M. (2018). An overview on application of machine learning techniques in optical networks. IEEE Communications Surveys \& Tutorials, 21(2), 1383-1408.

Nyarko-Boateng, O., Adekoya, A., \& Weyori, B. (2020a). Predicting the Actual Location of Faults in Underground Optical Networks using Linear Regression. Authorea.

Nyarko-Boateng, O., Adekoya, A. F., \& Weyori, B. A. (2020b). Using machine learning techniques to predict the cost of repairing hard failures in underground fiber optics networks. Journal of Big Data, 7(1), 1-16.

Potter, M. A., \& Jong, K. A. D. (2000). Cooperative Coevolution: An Architecture for Evolving Coadapted Subcomponents. Evolutionary Computation, 8(1), 1-29.

Pournoury, M., Moon, D. S., Nazari, T., Kassani, S. H., Do, M. H., Lee, Y. S., \& Oh, K. (2014). Low scattering loss fiber with segmented-core and depressed inner cladding structure. Optics Communications, 317, 13-17.

Rottondi, C., Barletta, L., Giusti, A., \& Tornatore, M. (2018). Machine-learning method for quality of transmission prediction of unestablished lightpaths. Journal of Optical Communications and Networking, 10(2), A286-A297. 
Rubio-Largo, A., Vega-Rodriguez, M. A., GomezPulido, J. A., \& Sanchez-Perez, J. M. (2012). A comparative study on multiobjective swarm intelligence for the routing and wavelength assignment problem. IEEE Transactions on Systems, Man and Cybernetics, Part C (Applications and Reviews), 42(6), 1644-1655.

Schmuck, H., Hehmann, J., Straub, M., \& Pfeiffer, T. (2006, September). Embedded OTDR techniques for cost-efficient fibre monitoring in optical access networks. In 2006 European Conference on Optical Communications (pp. 1-2). IEEE.

Seve, E., Pesic, J., Delezoide, C., Bigo, S., \& Pointurier, Y. (2018). Learning process for reducing uncertainties on network parameters and design margins. Journal of Optical Communications and Networking, 10(2), A298-A306.

Shahkarami, S., Musumeci, F., Cugini, F., \& Tornatore, M. (2018, March). Machine-learning-based softfailure detection and identification in optical networks. In 2018 Optical Fiber Communications Conference and Exposition (OFC) (pp. 1-3). IEEE.

Shiryaev, V. S., Karaksina, E. V., Churbanov, M. F., Kotereva, T. V., Stepanov, B. S., Ketkova, L. A., ... \& Antonov, I. N. (2018). Special pure germaniumrich Ga-Ge-As-Se glasses for active mid-IR fiber optics. Materials Research Bulletin, 107, 430-437.

Silva-Santos, C. H., Rodríguez-Esquerre, V. F., \& Hernández-Figueroa, H. E. (2015). Artificial Immune Network Design of Optical Multiplexers/Demultiplexers. Journal of Microwaves, Optoelectronics and Electromagnetic Applications, 14(2), 229-237.

Soujanya, A., Goud, O. S. C., Prasad, K. S., \& Reddy, G. P. (2017). Featured Based Pattern Analysis using Machine Learning and Artificial Intelligence Techniques for Multiple Featured Dataset. Materials Today: Proceedings, 4(8), 9039-9048.

Tamura, Y., Sakuma, H., Morita, K., Suzuki, M., Yamamoto, Y., Shimada, K., ... \& Hasegawa, T. (2018). The first $0.14-\mathrm{dB} / \mathrm{km}$ loss optical fiber and its impact on submarine transmission. Journal of Lightwave Technology, 36(1), 44-49.

Tanimura, T., Hoshida, T., Kato, T., Watanabe, S., \& Morikawa, H. (2018, March). Data-analytics-based optical performance monitoring technique for optical transport networks. In Optical Fiber Communication Conference (pp. Tu3E-3). Optical Society of America.

Tanimura, T., Hoshida, T., Rasmussen, J. C., Suzuki, M., \& Morikawa, H. (2016, July). OSNR monitoring by deep neural networks trained with asynchronously sampled data. In 2016 21st OptoElectronics and Communications Conference (OECC) held jointly with 2016 International Conference on Photonics in Switching (PS) (pp. 1-3). IEEE.
Temporão, G. P., de Faria, G. V., Urban, P. J., \& von der Weid, J. P. (2013, March). Fault location in passive optical networks using T-OTDR and wavelengthselective isolators. In National Fiber Optic Engineers Conference (pp. NM2I-4). Optical Society of America.

Thongdaeng, R., \& Worasucheep, D. R. (2016). Effect of bending radius and bending location on insertion loss in single mode fibers and polarization maintaining fibers. Procedia Computer Science, 86, 15-18.

Thrane, J., Wass, J., Piels, M., Diniz, J. C., Jones, R., \& Zibar, D. (2016). Machine learning techniques for optical performance monitoring from directly detected PDM-QAM signals. Journal of Lightwave Technology, 35(4), 868-875.

Urban, P. J., Getaneh, A., Von Der Weid, J. P., Temporão, G. P., Vall-llosera, G., \& Chen, J. (2013). Detection of fiber faults in passive optical networks. Journal of Optical Communications and Networking, 5(11), 1111-1121.

Vamsi, A. V. N., \& Rao, A. B. (2016). Fault Detection in Fiber Optic Communication Cable by Coherent Anti-Stokes Raman Scattering using Superconducting Nanowire Single-Photon Detector. Indian Journal of Science and Technology, 9, 1.

Vela, A. P., Ruiz, M., Fresi, F., Sambo, N., Cugini, F., Meloni, G., ... \& Castoldi, P. (2017). BER degradation detection and failure identification in elastic optical networks. Journal of Lightwave Technology, 35(21), 4595-4604.

Velmuzhov, A. P., Sukhanov, M. V., Shiryaev, V. S., Churbanov, M. F., Kotereva, T. V., Zernova, N. S., \& Fadeeva, D. A. (2018). Preparation of especially pure Ge-Se glasses via germanium monoselenide for Mid-IR fiber optics. Optical Materials, 84, 888-892.

Wang, D., Zhang, M., Fu, M., Cai, Z., Li, Z., Han, H., ... \& Luo, B. (2016). Nonlinearity mitigation using a machine learning detector based on $\$ \mathrm{k}$ \$-nearest neighbors. IEEE Photonics Technology Letters, 28(19), 2102-2105.

Wang, G., Mididoddi, C. K., Bai, F., Gibson, S., Su, L., Liu, J., \& Wang, C. (2018). UltraFast Optical Imaging using Multimode Fiber based Compressed Sensing and Photonic Time Stretch. arXiv preprint arXiv:1803.03061.

Wass, J., Thrane, J., Piels, M., Jones, R., \& Zibar, D. (2017, March). Gaussian process regression for WDM system performance prediction. In 2017 Optical Fiber Communications Conference and Exhibition (OFC) (pp. 1-3). IEEE.

Wilson, M. W. (2012). Location-based services, conspicuous mobility and the location-aware future. Geoforum, 43(6), 1266-12 
Wu, X., Jargon, J. A., Skoog, R. A., Paraschis, L., \& Willner, A. E. (2009). Applications of artificial neural networks in optical performance monitoring. Journal of Lightwave Technology, 27(16), 3580-3589.

Ye, H., Li, G. Y., \& Juang, B. H. (2017). Power of deep learning for channel estimation and signal detection in OFDM systems. IEEE Wireless Communications Letters, 7(1), 114-117.

Yuksel, K., Moeyaert, V., Wuilpart, M., \& Mégret, P. (2008, June). Optical layer monitoring in passive optical networks (PONs): A review. In 2008 10th Anniversary International Conference on Transparent Optical Networks (Vol. 1, pp. 92-98). IEEE.

Zeng, H., \& Huang, C. (2004, November). Fault detection and path performance monitoring in meshed all-optical networks. In IEEE Global Telecommunications Conference, 2004. GLOBECOM'04. (Vol. 3, pp. 2014-2018). IEEE.

Zhang, X., Lu, F., Chen, S., Zhao, X., Zhu, M., \& Sun, $X$. (2016). Remote coding scheme based on waveguide Bragg grating in PLC splitter chip for PON monitoring. Optics express, 24(5), 4351-4364.
Zhao, Y., Li, X., Li, H., Wang, X., Zhang, J., \& Huang, S. (2013a). Multi-link faults localization and restoration based on fuzzy fault set for dynamic optical networks. Optics express, 21(2), 1496-1511.

Zhao, T., Wang, A., Wang, Y., Zhang, M., Chang, X., Xiong, L., \& Hao, Y. (2013b). Fiber fault location utilizing traffic signal in optical network. Optics express, 21(20), 23978-23984.

Zhi, W., Guobin, R., Shuqin, L., \& Shuisheng, J. (2003). Loss properties due to Rayleigh scattering in different types of fiber. Optics express, 11(1), 39-47.

Zhou, X., Chen, Z., Zhou, H., \& Hou, J. (2014). Modefield adaptor between large-mode-area fiber and single-mode fiber based on fiber tapering and thermally expanded core technique. Applied optics, 53(22), 5053-5057.

Zibar, D., Piels, M., Jones, R., \& Schäeffer, C. G. (2015). Machine learning techniques in optical communication. Journal of Lightwave Technology, 34(6), 1442-1452.

Zou, N., Namihira, Y., Ndiaye, C., \& Ito, H. (2007, March). Fault location for branched optical fiber networks based on OFDR technique using FSF laser as light source. In National Fiber Optic Engineers Conference (p. NWC2). Optical Society of America. 